Research article

Open Access

\title{
Expression of novel extracellular sulfatases Sulf-1 and Sulf-2 in normal and osteoarthritic articular cartilage
}

\author{
Shuhei Otsuki ${ }^{1}$, Noboru Taniguchi ${ }^{1}$, Shawn P Grogan ${ }^{1}$, Darryl D'Lima ${ }^{1}$, Mitsuo Kinoshita ${ }^{2}$ and \\ Martin Lotz 1
}

\author{
1Division of Arthritis Research, The Scripps Research Institute, 10550 North Torrey Pines Road, La Jolla, CA 92037, USA \\ 2Department of Orthopedic Surgery, Osaka Medical College, 2-7 Daigaku-machi Takatsuki 569-8686, Osaka, Japan \\ Corresponding author: Shuhei Otsuki, otsuki@scripps.edu \\ Received: 14 Jan 2008 Revisions requested: 18 Feb 2008 Revisions received: 4 Apr 2008 Accepted: 28 May 2008 Published: 28 May 2008 \\ Arthritis Research \& Therapy 2008, 10:R61 (doi:10.1186/ar2432) \\ This article is online at: http://arthritis-research.com/content/10/3/R61 \\ (c) 2008 Otsuki et al.; licensee BioMed Central Ltd. \\ This is an open access article distributed under the terms of the Creative Commons Attribution License (http://creativecommons.org/licenses/by/2.0), \\ which permits unrestricted use, distribution, and reproduction in any medium, provided the original work is properly cited.
}

\begin{abstract}
Introduction Changes in sulfation of cartilage glycosaminoglycans as mediated by sulfatases can regulate growth factor signaling. The aim of this study was to analyze expression patterns of recently identified extracellular sulfatases Sulf- 1 and Sulf- 2 in articular cartilage and chondrocytes.

Methods Sulf-1 and Sulf-2 expressions in human articular cartilage from normal donors and patients with osteoarthritis (OA) and in normal and aged mouse joints were analyzed by real-time polymerase chain reaction, immunohistochemistry, and Western blotting.

Results In normal articular cartilage, Sulf- 1 and Sulf- 2 mRNAs and proteins were expressed predominantly in the superficial zone. OA cartilage showed significantly higher Sulf-1 and Sulf-

2 mRNA expression as compared with normal human articular cartilage. Sulf protein expression in OA cartilage was prominent in the cell clusters. Western blotting revealed a profound increase in Sulf protein levels in human OA cartilage. In normal mouse joints, Sulf expression was similar to human cartilage, and with increasing age, there was a marked upregulation of Sulf.

Conclusion The results show low levels of Sulf expression, restricted to the superficial zone in normal articular cartilage. Sulf mRNA and protein levels are increased in aging and OA cartilage. This increased Sulf expression may change the sulfation patterns of heparan sulfate proteoglycans and growth factor activities and thus contribute to abnormal chondrocyte activation and cartilage degradation in OA.
\end{abstract}

\section{Introduction}

Osteoarthritis $(\mathrm{OA})$ is the most prevalent joint disease and is characterized by degradation of articular cartilage, subchondral bone remodeling, and joint inflammation $[1,2]$. Chondrocytes in OA cartilage are activated by cytokines and growth factors $[3,4]$ to a catabolic phenotype that leads to progressive extracellular matrix (ECM) destruction and abnormal chondrocyte differentiation [4,5]. Cartilage ECM consists of collagens, glycoproteins, proteoglycans, and glycosaminoglycans (GAGs). The major GAGs in cartilage are hyaluronic acid, chondroitin sulfate, keratan sulfate, dermatan sulfate, and heparan sulfate. GAGs were previously shown to be important determinants of cartilage biomechanical properties but also have recently been shown to bind and regulate the activity of several cytokines and growth factors. In particular, the sulfation patterns of GAGs are critical in determining the binding capacity and specificity for cytokines and growth factors [6-9]. Heparan sulfate proteoglycans (HSPGs) also act as co-receptors for heparin-binding growth factors and cytokines [10]. The sulfation of heparan sulfate residues is required for interactions with heparin-binding factors that are also know to be important regulators of chondrocytes, including Wnt, fibroblast growth factor (FGF), vascular endothelial growth factor (VEGF), and bone morphogenetic proteins (BMPs) [11-14].

Sulfotransferases and sulfatases establish GAG sulfation in the endoplasmatic reticulum and Golgi network prior to

$\mathrm{BMP}=$ bone morphogenetic protein; $\mathrm{bp}=$ base pairs; $\mathrm{DMEM}=$ Dulbecco's modified Eagle's medium; $\mathrm{ECM}=$ extracellular matrix; FGF = fibroblast growth factor; GAG = glycosaminoglycan; GAPDH = glyceraldehyde-3-phosphate dehydrogenase; HSPG = heparan sulfate proteoglycan; OA = osteoarthritis; $\mathrm{PCR}=$ polymerase chain reaction; $\mathrm{PM}=$ pericellular matrix; $\mathrm{RT}-\mathrm{PCR}=$ reverse transcription-polymerase chain reaction; Sulf $=$ heparan sulfate 6 -O endosulfatase; TBST $=$ Tris-buffered saline-Tween; TM = temporomandibular. 
secretion [15]. Classic sulfatases are intracellular enzymes that cleave sulfate esters from substrates that range from small cytosolic steroids, such as estrogen sulfate, to complex cell surface carbohydrates, such as the GAGs [15]. A novel class of extracellular heparan sulfate 6-O endosulfatase (Sulf) has recently been identified and in mammalians includes two isoforms, Sulf-1 and Sulf-2 [16-18]. These enzymes exist in a cell surface-associated and soluble form and hydrolyze the 6-O sulfate of HSPGs $[17,19]$. Most of the current information on Sulf-1 and Sulf-2 is related to cancer and development [2023]. Specifically, 6-O sulfation of heparan sulfate is required for receptor dimerization and FGF signaling while 6-O desulfation is associated with reduced FGF2 signaling [24]. Sulf-1 also regulates Wnt signaling through desulfation of cell surface HSPGs [16].

OA is associated with changes in GAG expression levels and sulfation patterns $[6,8,9]$, but mechanisms and consequences remain to be analyzed. This study addresses the hypothesis that the novel extracellular sulfatases may be involved in regulating the growth factor signaling balance in articular cartilage. The results show that Sulf-1 and Sulf-2 are (a) expressed in human articular cartilage, and (b) are preferentially expressed in the superficial zone and that (c) their expression is altered in osteoarthritic and aging cartilage.

\section{Materials and methods Cartilage procurement and processing}

All tissue samples were graded according to a modified Mankin scale [25], with a score of less than 3 points being normal and a score of greater than 5 representing OA [26]. Normal articular cartilage was harvested from femoral condyles and tibial plateaus of human tissue donors under approval of the Scripps Human Subjects Committee. Osteoarthritic cartilage was obtained from patients undergoing knee replacement surgery. The thickness of these cartilages ranged from 1.5 to $2.8 \mathrm{~mm}$. Once cartilage surfaces were rinsed with saline, scalpels were used to cut parallel sections $5 \mathrm{~mm}$ apart, vertically from the cartilage surface onto the subchondral bone. These cartilage strips were then resected from the bone. Human chondrocytes were isolated and cultured as described previously [27]. The cartilage tissue was incubated with trypsin at $37^{\circ} \mathrm{C}$ for 10 minutes. After the trypsin solution was removed, the tissue slices were treated for 12 to 16 hours with type IV clostridial collagenase in Dulbecco's modified Eagle's medium (DMEM) with 5\% fetal calf serum. After initial isolation, the cells were kept in high-density cultures in DMEM (high glucose) supplemented with 10\% CS, L-glutamine, and antibiotics and allowed to attach to the surface of the culture flasks. After the cells had grown to confluence, they were split once (passage 1) and grown to confluence again for use in the experiments.
RNA isolation from cartilage and cultured chondrocytes RNA was isolated from fresh frozen cartilage by homogenizing the tissue in a freezer mill (Spex CertiPrep, Inc., Metuchen, NJ, USA) and extracting the homogenate in Trizol (Life Technologies, Inc., now part of Invitrogen Corporation, Carlsbad, CA, USA). The samples were extracted with chloroform and centrifuged at $15,000 \mathrm{~g}$ for 20 minutes, and the aqueous phase was collected. An equal volume of $70 \%$ ethanol was added, mixed, and applied to RNeasy columns (Qiagen Inc., Valencia, CA, USA). RNA concentrations were determined using RiboGreen reagent (Molecular Probes Inc., now part of Invitrogen Corporation). Total RNA was isolated from chondrocyte cultures plated at confluence at $3 \times 10^{6}$ cells per $100-\mathrm{mm}$ plate using the RNeasy kit (Qiagen Inc.) with on-column DNA digestion. Complementary DNA was produced using the SuperScript III First-Strand kit (Invitrogen Corporation) with random hexamers.

\section{Quantitative polymerase chain reaction for Sulf-1 and Sulf-2}

Sulf-1 and Sulf-2 primers and conditions for reverse transcription-polymerase chain reaction (RT-PCR) were based on the protocol of Morimoto-Tomita and colleagues [17]. Real-time RT-PCR with SYBR green detection was performed using an iCycler (Bio-Rad Laboratories, Inc., Hercules, CA, USA) as follows: 2 minutes at $50^{\circ} \mathrm{C}$ and then 10 minutes at $95^{\circ} \mathrm{C}$ for initial denaturation, followed by 40 cycles at $95^{\circ} \mathrm{C}$ (15 seconds), $60^{\circ} \mathrm{C}$ ( 1 minute), followed by the measurement of fluorescence at the end of each cycle. Each run included a melting curve to determine the correct response of the primers [28]. The following primers were used: Sulf-1: forward 5'-AGACCTAAGAAT CTTGATGTTGGAA-3', reverse 5'-CCATCCCATAACTGTCCTCTG-3'(74 base pairs [bp], NM15170), Sulf-2: forward 5'-TGAGGGAAGTCCGAGGTCAC-3', reverse 5'-CTTGCGGAGTTTCTTCTTGC-3' (194 bp, NM018837, NM198596), glyceraldehyde-3-phosphate dehydrogenase (GAPDH): forward 5'-ACCCACTCCTCCACCTTTGA-3', reverse 5'-ATGAGGTCCACCACCCTGTT-3'.

Primers were selected in consideration of the low homology between the sequences of Sulf-1 and Sulf-2. Furthermore, human Sulf-2 primers were designed to detect both Sulf-2 splice variants, NM 018837 and NM 198596. The specificity of detection of Sulf-1 and Sulf- 2 was confirmed by sequencing the PCR products after isolation with the QIAquick gel extraction kit (Qiagen Inc.). Changes in Sulf gene expression were calculated relative to GAPDH.

\section{Histology and immunohistochemistry}

Cartilage tissues were fixed with $4 \%$ paraformaldehyde and stained with safranin $O$. Sulf antibodies were purchased from Abcam Inc. (Cambridge, MA, USA). Paraffin-fixed samples were first deparaffinized in xylene substitute Pro-Par Clearant (Anatech Ltd., Battle Creek, MI, USA), ethanol then water for rehydration. After washing with PBS, sections were blocked 
with $0.1 \%$ Tween 20 with 3\% normal goat serum for 30 minutes at room temperature. Sulf- 1 and Sulf- 2 antibodies $(2 \mu \mathrm{g} /$ $\mathrm{mL})$ and normal mouse $\mathrm{lgG}(1 \mu \mathrm{g} / \mathrm{mL})$ as negative control were applied and incubated overnight at $4^{\circ} \mathrm{C}$. After washing with PBS, sections were incubated with biotinylated goat antimouse secondary antibody for 30 minutes (1:200; Vector Laboratories Inc., Burlingame, CA, USA) and then incubated with Vectastain ABC-AP kit (AK-5000; Vector Laboratories Inc.) for 30 minutes at room temperature. Finally, sections were stained with an alkaline phosphatase substrate kit (Vector Laboratories Inc.).

\section{Quantification and localization of signals throughout cartilage}

Sulf-1 and Sulf-2 localization throughout each cartilage zone was assessed systematically by counting positive and negative cells in a $50 \times 50 \mu \mathrm{m}$ grid (using a $\times 40$ field objective) starting from the cartilage surface to the deep zone. This was repeated a minimum of five times for each section. The identification of each zone was based on previously reported characteristics that comprise cell shape, morphology, orientation, and pericellular matrix (PM) deposition [29]. Thus, superficial zone (SZ) cells were characterized by their elongated shape, their parallel orientation relative to the surface, and lack of extensive PM. These cells predominate within the first $50 \mu \mathrm{m}$. The middle zone $(\mathrm{MZ})$ was distinguishable by rounded cells that did not exhibit an organized orientation relative to the surface, that have ECM rich in proteoglycans, and that show the presence of PM. Conversely, deep zone (DZ) cells were recognized with an extensive PM deposition and organized in columns of chondron groups of three or more cells. The depth of each zone was recorded for each section for comparative analysis on the frequency of positive signal in each zone. The frequency of positive cells was expressed as a percentage relative to the total number of cells counted in each zone.

\section{Western blotting}

Cartilage was cut into 1 -mm-thin slices, and 200 to $1,000 \mathrm{mg}$ of frozen cartilage was pulverized in a liquid nitrogen-cooled freezer mill for two cycles of 1.5 minutes at the rate of maximum impact frequency. Dry weight of normal and OA cartilage was measured and the same amount of protein was resuspended in SDS gel loading buffer (50 mM Tris $\mathrm{pH} 6.8,10 \%$ glycerol, 4\% sodium dodecyl sulfate, 10\% 2-mercaptoethanol, and $0.001 \%$ bromophenol blue) and mixed for 2 hours at room temperature. Centrifugation at $14,000 \mathrm{rpm}$ was performed for 30 minutes and then supernatants were harvested and heated at $80^{\circ} \mathrm{C}$ for 10 minutes. The concentrated samples were then adjusted for equal volumes before resolution on $12 \%$ Tris-Glycine gels (Invitrogen Corporation). Protein was transferred to nitrocellulose membranes (Invitrogen Corporation), blocked with 5\% dry milk in Tris-buffered saline-Tween (TBST), and blotted with mouse polyclonal antibody specific for Sulf-1 or Sulf-2 (Abcam Inc.) for 1 hour. The membranes were then incubated with horseradish peroxidase-conjugated anti- mouse IgG (Santa Cruz Biotechnology, Inc., Santa Cruz, CA, USA) for 1 hour. Afterwards, the membranes were washed three times with TBST and developed using the enhanced chemiluminescent substrate from Pierce (Rockford, IL, USA).

\section{Analysis of murine joints}

All animal experiments were performed according to protocols approved by the Institutional Animal Care and Use Committee at The Scripps Research Institute (La Jolla, CA, USA). Sulf-1 and Sulf-2 expression was analyzed by immunohistochemistry in temporomandibular (TM) joints and knee joints of 1-, 6-, 9-, and 12-month-old C57BL/6J mice. Each mouse joint was cut in half along the mid-sagittal plane and fixed in 10\% zinc-buffered formalin (Z-Fix; Anatech Ltd.) for 2 to 3 days and then decalcified in Shandon TBD-2 decalcifier (Fisher Scientific Pittsburgh, PA, USA) for 2 to 3 weeks. Three-millimeter serial sections (from posterior to anterior) were cut and immunostained for Sulf- 1 and Sulf- 2 as described above.

\section{Statistical analysis}

Statistically significant differences between two groups were determined with $t$ tests. The results are reported as mean \pm standard deviation. $P$ values of less than 0.05 were considered significant.

\section{Results}

\section{Sulf gene expression in articular cartilage}

Sulf- 1 and Sulf- 2 mRNA expression in eight OA donors (49 to 68 years old; Mankin score: 7 to 10 points) was significantly higher than in eight young donors (19 to 37 years old; Mankin score: 0 to 2 points) as determined by real-time PCR (Figure 1).

\section{Localization of Sulf-1 and Sulf-2 proteins in human articular cartilage}

Young and old normal samples as seen on safranin $O$ staining (Figure 2a, d, g) had only a few Sulf-positive cells in the super-

\section{Figure 1}

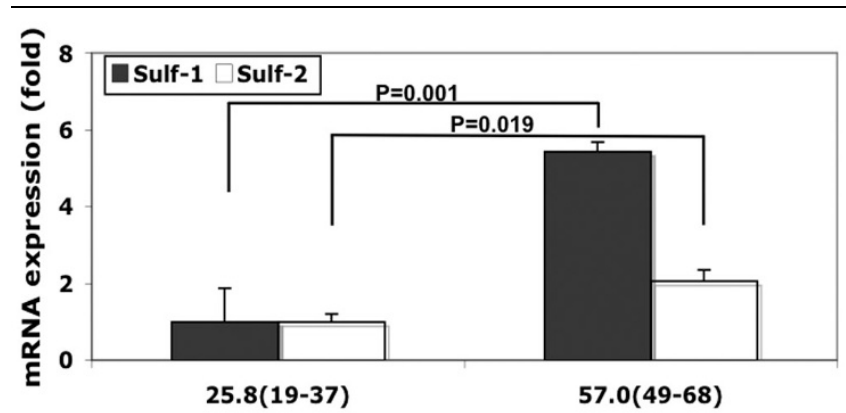

Sulf mRNA expression in normal and osteoarthritis (OA) cartilage. Sulf1 and Sulf-2 mRNA expression in articular cartilage were determined by quantitative polymerase chain reaction in eight normal (mean age: 20.3 years, range: 19 to 37 years; Mankin score: 0 to 2 points) and eight OA (mean age: 57 years, range: 49 to 68 years; Mankin score: 7 to 10 points) donors. Both Sulf-1 and Sulf-2 expression were significantly higher in the OA group (Sulf-1: $P=0.001$, Sulf-2: $P=0.019$ ). 
ficial zone (Figure 2b, c, e, f, h, i) and no positive cells in the middle and deep zones. In general, the expression of Sulf-2 appeared more intense than Sulf-1 in normal cartilage. In OA cartilage, many positive cells were detected, especially in chondrocyte clusters (Figure $3 g, h, k, l)$. The representative example of 65-year-old cartilage had both normal areas (Mankin score: 2) (Figure $3 a, b$ ) and $\mathrm{OA}$ areas with fibrillations and cluster formation (Mankin score: 8) (Figure 3c, d). The normal appearing areas from OA joints had $18.5 \%$ Sulf-1-positive and $31.9 \%$ Sulf-2-positive cells in the superficial zone (Figure $3 e, f, i, j$ ), which was greater than in normal cartilage (Figure 2). On the other hand, OA areas had $75.3 \%$ Sulf-1-positive and 73.2\% Sulf-2-positive cells (Figure $3 g, h, k, l$ ).

Figure 4 shows quantitative analysis of the zonal distribution of Sulf-1- and Sulf-2-expressing cells in eight normal (17 to 37 years old) and eight OA (43 to 82 years old) donors. In OA, the superficial zone was already eroded. The middle zone in OA cartilage had significantly more positive cells than normal $\left({ }^{\star} P<0.01\right)$. Moreover, the number of Sulf-2-positive cells in the superficial and middle zones was greater than Sulf-1expressing cells $(P=0.02)$.

Western blotting was performed to visualize Sulf proteins and determine differences in the expression between normal and

Figure 2

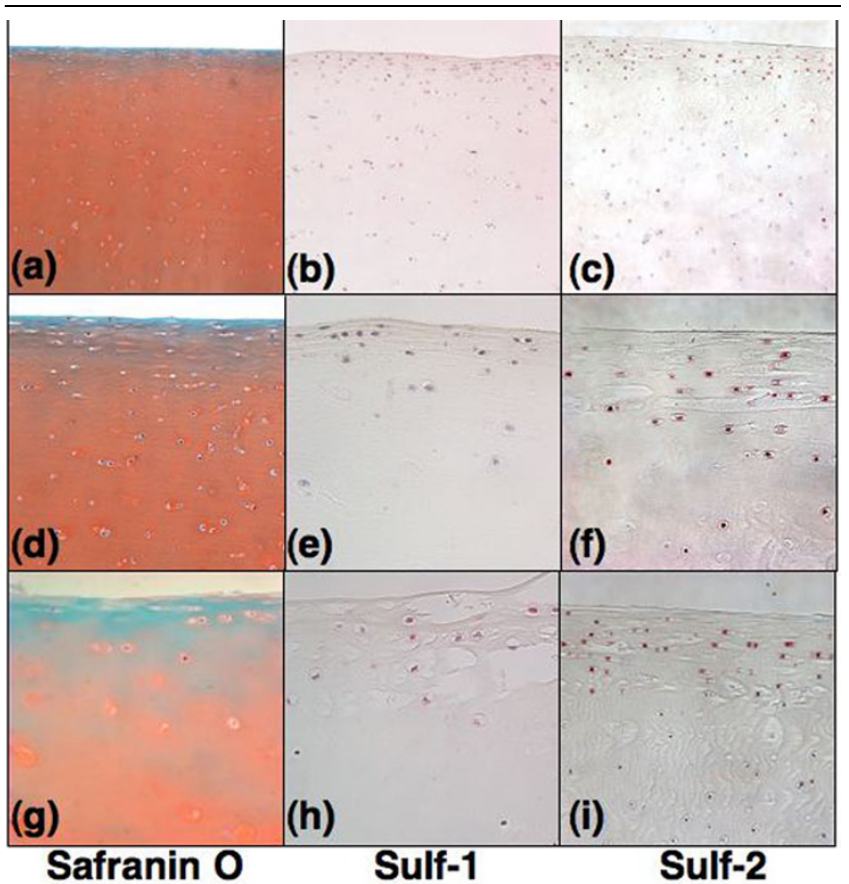

Localization of Sulf-1 and Sulf- 2 in normal cartilage. Representative sections of 26-year-old (a, d) and 74-year-old (g) normal cartilage (Mankin scores: 0 and 2) as seen on safranin O staining are shown $(n=$ $8 ; 19$ to 37 years old). Sulf-positive cells (brown staining) are present in the superficial zone and the top of the middle zone, and Sulf-2 expression is greater than Sulf-1 (b, c, e, f, h, i) in both young and old cartilage. Magnifications: $\times 10(\mathrm{a}-\mathrm{c})$ and $\times 40(\mathrm{~d}-\mathrm{i})$.
OA. In total protein extracts from normal cartilage, Sulf- 1 and Sulf-2 were not detectable. In contrast, high levels of Sulf-1 and Sulf-2 protein were detected in OA cartilage (Figure 5). The major Sulf- 1 and Sulf-2 protein bands migrated at approximately $72 \mathrm{kDa}$, which is the molecular mass of the secreted proteins $[17,30,31]$.

\section{Sulf-1 and Sulf-2 expression in murine joints}

TM joints from normal C57BL/6J mice $(n=6)$ were analyzed with safranin $\mathrm{O}$ staining (Figure $6 \mathrm{a}-\mathrm{C}$ ) and immunohistochemistry for Sulf-1 and Sulf-2 (Figure 6d-i). Histology showed thinning and reduced cell density in articular cartilage with increasing age (Figure 6c). In 6-month-old mice, only a few cells were positive for Sulf-1 but Sulf-2-positive cells were present throughout the cartilage. There was a marked increase in Sulf-2 expression at 9 months and in Sulf- 1 expression at 12 months. In murine knee joints $(n=8)$, there was high Sulf expression at 1 month of age, followed by a decrease with joint maturation. Increased expression of Sulf- 1 and Sulf- 2 was seen in the articular cartilage of murine knee joints by 12 months of age, when early OA-like changes become apparent (Figure 7).

\section{Discussion}

Chondrocytes in osteoarthritic cartilage are activated by cytokines, growth factors, and mechanical stress to produce matrix-degrading enzymes and pro-inflammatory cytokines with an overall shift from anabolic to catabolic responses $[32,33]$. Besides control of gene expression, protein synthesis, and secretion, the biological activity of cytokines and growth factors is regulated by binding to ECM proteins such as GAGs [34,35]. The sulfation pattern of HSPGs has recently been shown to be critical for determining the specificity and affinity of binding to growth factors and morphogens [36]. The sulfation patterns of HSPGs are determined during intracellular biosynthesis and can be further modified on cell surface-associated and extracellular HSPGs by a novel class of extracellular sulfatases which includes two enzymes, Sulf-1 and Sulf-2. Previously, Sulf-1 and Sulf-2 mRNAs were shown to be expressed at high levels in regions of developing cartilage and bone [37]. The present study reports on the expression of Sulf in mature cartilage and changes with aging and OA.

In normal articular cartilage, Sulf-1 and Sulf-2-positive cells were predominantly localized in the superficial zone and Sulf2 was more highly expressed than Sulf- 1 . This observation adds further to the zone-specific differences of chondrocyte subsets, in particular of the superficial zone cells [38-40].

OA cartilage showed higher expression of Sulf- 1 and Sulf- 2 as compared with normal tissue in all experimental approaches used in the present study, including quantitative PCR on cartilage and cultured chondrocytes, immunohistochemistry, and Western blotting. Aging and OA are closely linked. To address 


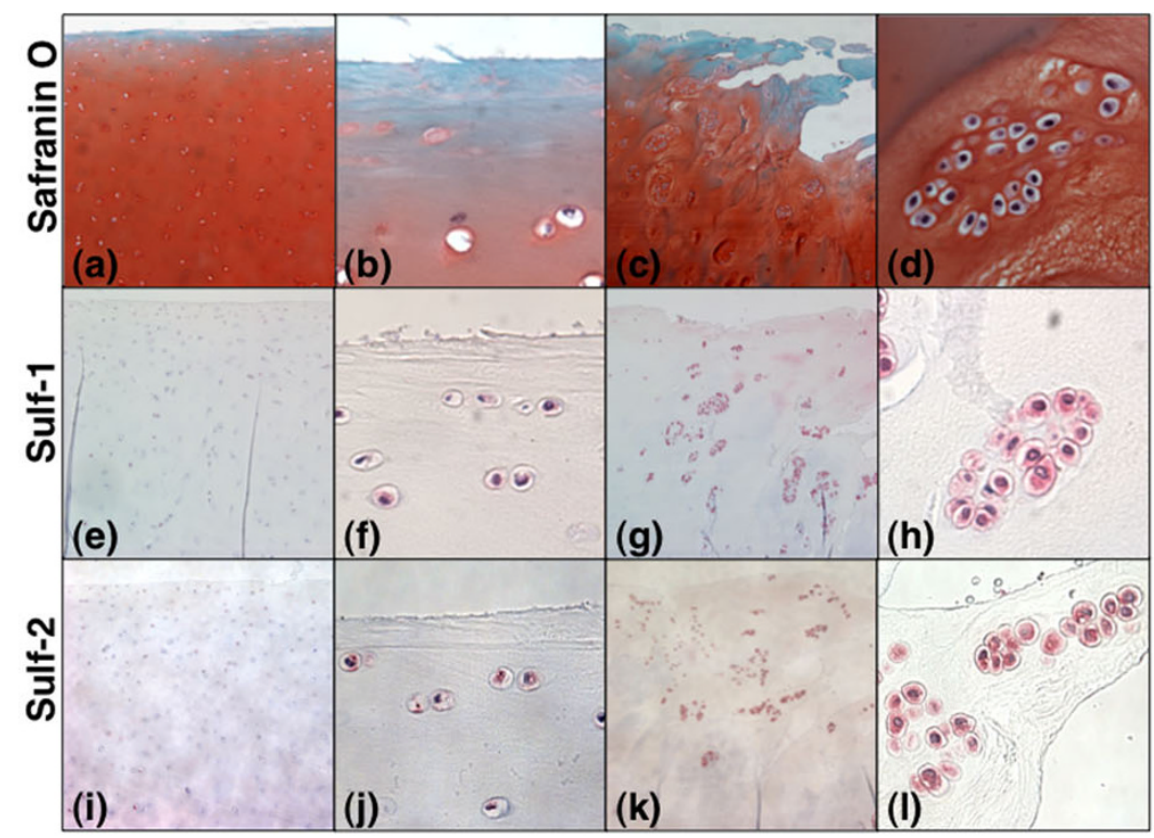

Localization of Sulf-1 and Sulf-2 in normal-appearing and fibrillated cartilage from the same osteoarthritis (OA) donor. Sulf localization was determined with 16 donors (19 to 82 years old). Cartilage from a representative 65-year-old donor had both normal areas (Mankin score: 2 ) and OA areas (Mankin score: 8). Sulf-positive cells were more frequent in OA areas than in normal-appearing cartilage. Magnifications: $\times 10$ (a, c, e, g, i, k) and $\times 100(b, d, f, h, j, l)$.

the influence of these variables on Sulf expression, we analyzed normal-appearing and fibrillated cartilages in the same joints from patients with OA. In the OA joints, Sulf expression was higher in the fibrillated areas. Even in areas that had almost normal surface layers and safranin $O$ staining patterns, Sulf expression was higher than in normal cartilage from young healthy donors. The TM joint is an important growth and artic- ulation center in the craniofacial complex, and with aging, it develops spontaneous degenerative OA lesions [41]. TM joints showed strongly increased Sulf expression between 6 and 12 months of age, when cartilage thickness and cellularity were reduced, but fibrillations had not yet developed. Sulf expression was also determined in murine knee joints. Interestingly, Sulf expression was high at 1 month of age and

Figure 4

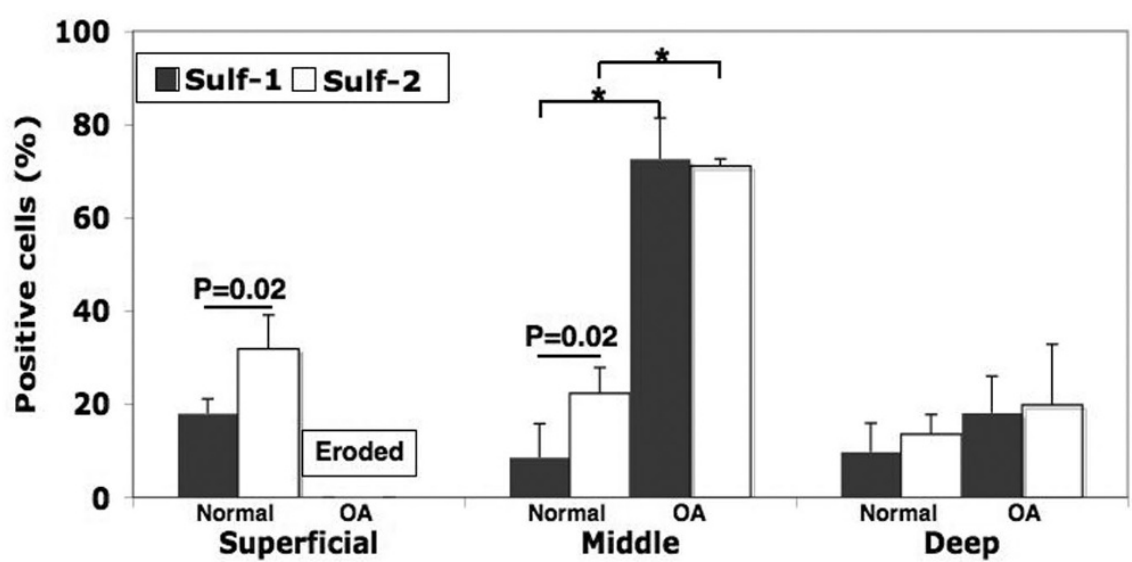

Sulf-1 and Sulf-2 expression in specific zones of normal and osteoarthritis (OA) cartilage. The number of Sulf-1- or Sulf-2-positive cells was counted in the superficial, middle, and deep zones of sections from normal $(n=8)$ and OA $(n=8)$ cartilage that were stained with specific antibodies. In normal cartilage, the percentage of Sulf-2-positive cells was highest in the superficial zone. The superficial zone in OA cartilage was eroded. The OA middle zone had significantly more Sulf-1-positive cells than the other zones $\left({ }^{\star} P<0.01\right)$. Sulf-2 expression in normal cartilage was significantly higher than Sulf-1 $(P=0.02)$. 
Figure 5

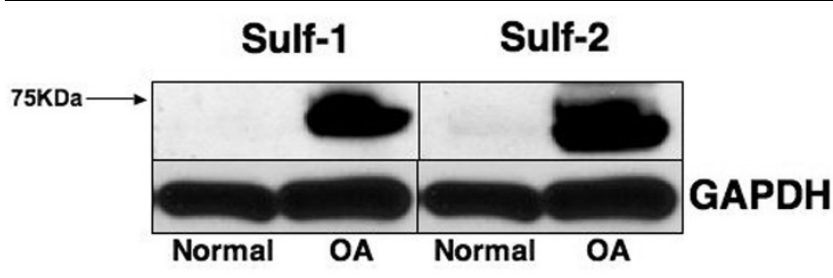

Sulf-1 and Sulf-2 protein expression in normal and osteoarthritis (OA) cartilage. Sulf protein expression in normal (43 years old; Mankin score: 2) and OA (79 years old; Mankin score: 9) cartilage. Immunoblottings of Sulf-1, Sulf-2, and GAPDH (glyceraldehyde-3-phosphate dehydrogenase) were performed on protein extracts from normal and OA cartilage.

decreased with joint maturation, suggesting a role in this process. By 12 months of age, Sulf expression increased again with the simultaneous development of OA-like changes. Taken together, these findings from human and murine joints indicate that Sulfs are upregulated with age and at early stages of the matrix degradation process.

The present observations of increased Sulf expression suggest a role in OA pathogenesis. Altered GAG sulfation patterns on chondroitin sulfate and dermatan sulfate have been reported in aging and $\mathrm{OA}[9,42]$, but changes in heparan sulfation patterns under these conditions have not yet been analyzed. The HSPGs are potential targets of Sulf [19], and their expression in articular cartilage and changes in OA have been

\section{Figure 6}

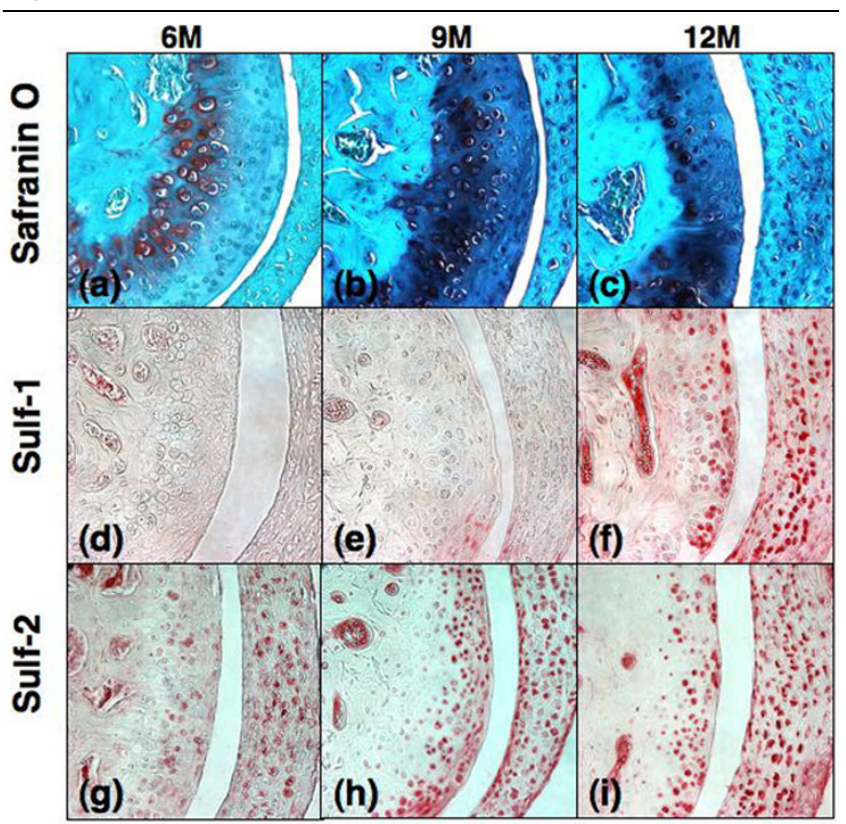

Sulf-1 and Sulf-2 expression in murine temporomandibular joints. Safranin O staining (a-c) and immunohistochemistry (d-i) were performed on sections from temporomandibular joints of $\mathrm{C} 57 \mathrm{BL} / 6 \mathrm{~J}$ mice at 6,9 , and 12 months of age $(n=6)$. Magnification: $\times 40$. demonstrated in several previous publications on syndecan [43-49], perlecan [50-54], and glypican [44,54]. In particular, syndecan-1, syndecan-3 $[45,46]$, and perlecan [50] are overexpressed in severe OA. Furthermore, some of these studies have shown that HSPGs are overexpressed, specifically in cell clusters in OA cartilage. In this study, we also showed Sulf-1 and Sulf-2 overexpression in OA cartilage, particularly in clusters. Collectively, this information documents the presence of the HSPGs that are the major known sulfatase substrates in articular cartilage. In addition, there appears to be similar expression of the enzymes and substrates in OA-affected cartilage. Changes in sulfation of heparan sulfate are important in cell behavior and organogenesis [55] and affect several growth factor signaling pathways. 6-O sulfated heparan sulfates are required for FGF receptor dimerization. Sulf-1 desulfates cell surface heparan sulfate and inhibits FGF signaling [24,56]. Im and colleagues [57] showed that FGF2 induced matrix metalloproteinase-13 in articular chondrocyte and contributes to OA progression. FGF2 may regulate Sulf expression and maintain the anabolic and catabolic balance in cartilage.

Sulf-1 also mediates 6-O desulfation of the heparan sulfateWnt complex so that it interacts with Frizzled receptor, initiating Wnt target gene expression [19]. Wnt signaling is important in cartilage. Wht and $\beta$-catenin activation are associated with inhibition of type II collagen expression [58] with GAG loss [8] and abnormal chondrocyte differentiation in OA [59]. Thus, Wnt signaling, activated by Sulf, may accelerate the progression of OA. Sulf-1 regulates BMP signaling, which is important in cartilage homeostasis. The BMP antagonist Noggin is a heparin-binding protein that is associated with the cell surface through HSPGs, where it inhibits BMP signaling. Sulf-1 desulfates heparan sulfate, releases Noggin, and thus restoring BMP signaling [11].

\section{Conclusion}

This study is the first to show increased Sulf expression in OA cartilage. Sulf-1 and Sulf-2 are highly expressed in OA cartilage, especially in clusters and even in normal-appearing cartilage in OA joints. The ability of Sulfs to regulate growth factor pathways (such as FGF, Wnt, or BMP) that are important in cartilage suggests that their overexpression in OA contributes to the abnormal chondrocyte activation and ECM degradation. Inhibition of Sulfs may represent a new approach to correct these pathogenetic processes.

\section{Competing interests}

The authors declare that they have no competing interests.

\section{Authors' contributions}

SO carried out the experimental work, performed the statistical analysis, and drafted the manuscript. NT and SG performed experimental work and helped to draft the manuscript. DD'L and $M L$ analyzed the data. $M L$ designed and 

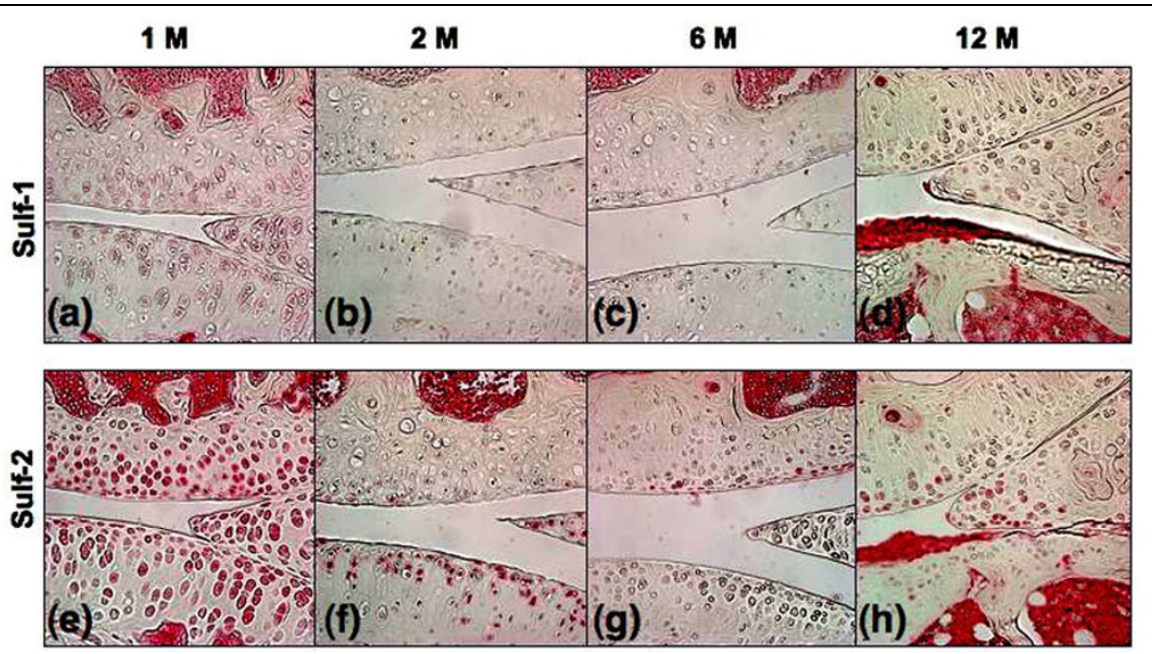

Sulf-1 and Sulf-2 expression in murine knee joints. Immunohistochemistry for Sulf-1 (a-d) and Sulf-2 (e-h) was performed on sections from knee joints of C57BL/6J mice at 1, 2, 6, and 12 months of age $(n=8)$. Magnification: $\times 40$.

organized the study and drafted the manuscript. All authors read and approved the final manuscript.

\section{Acknowledgements}

This study was supported by NIH grant AG07996. We thank Diana C Brinson, Lilo Creighton, and Jean Valbracht for their excellent technical support.

\section{References}

1. Glass GG: Osteoarthritis. Dis Mon 2006, 52:343-362.

2. Felson DT, Neogi T: Osteoarthritis: is it a disease of cartilage or of bone? Arthritis Rheum 2004, 50:341-344.

3. Goldring MB: The role of the chondrocyte in osteoarthritis. Arthritis Rheum 2000, 43:1916-1926.

4. Lotz M: Cytokines in cartilage injury and repair. Clin Orthop Relat Res 2001, 391(Suppl):S108-115.

5. Sandell $L J$, Aigner T: Articular cartilage and changes in arthritis. An introduction: cell biology of osteoarthritis. Arthritis research 2001, 3:107-113.

6. Sauerland K, Plaas AH, Raiss RX, Steinmeyer J: The sulfation pattern of chondroitin sulfate from articular cartilage explants in response to mechanical loading. Biochim Biophys Acta 2003, 1638:241-248.

7. Burkhardt D, Michel BA, Baici A, Kissling R, Theiler R: Comparison of chondroitin sulphate composition of femoral head articular cartilage from patients with femoral neck fractures and osteoarthritis and controls. Rheumatol Int 1995, 14:235-241.

8. Shortkroff S, Yates KE: Alteration of matrix glycosaminoglycans diminishes articular chondrocytes' response to a canonical Wnt signal. Osteoarthritis Cartilage 2007, 15:147-154.

9. Bayliss MT, Osborne D, Woodhouse S, Davidson C: Sulfation of chondroitin sulfate in human articular cartilage. The effect of age, topographical position, and zone of cartilage on tissue composition. J Biol Chem 1999, 274:15892-15900.

10. Bishop JR, Schuksz M, Esko JD: Heparan sulphate proteoglycans fine-tune mammalian physiology. Nature 2007, 446:1030-1037.

11. Viviano BL, Paine-Saunders S, Gasiunas N, Gallagher J, Saunders S: Domain-specific modification of heparan sulfate by Qsulf1 modulates the binding of the bone morphogenetic protein antagonist Noggin. J Biol Chem 2004, 279:5604-5611.

12. Lin X, Perrimon N: Dally cooperates with Drosophila Frizzled 2 to transduce Wingless signalling. Nature 1999, 400:281-284.

13. Lin X, Buff EM, Perrimon N, Michelson AM: Heparan sulfate proteoglycans are essential for FGF receptor signaling during
Drosophila embryonic development. Development 1999, 126:3715-3723.

14. Mitsi M, Hong Z, Costello CE, Nugent MA: Heparin-mediated conformational changes in fibronectin expose vascular endothelial growth factor binding sites. Biochemistry 2006, 45:10319-10328.

15. Hanson SR, Best MD, Wong $\mathrm{CH}$ : Sulfatases: structure, mechanism, biological activity, inhibition, and synthetic utility. Angew Chem Int Ed Eng/ 2004, 43:5736-5763.

16. Dhoot GK, Gustafsson MK, Ai X, Sun W, Standiford DM, Emerson CP Jr: Regulation of Wnt signaling and embryo patterning by an extracellular sulfatase. Science 2001, 293:1663-1666.

17. Morimoto-Tomita M, Uchimura K, Werb Z, Hemmerich S, Rosen SD: Cloning and characterization of two extracellular heparindegrading endosulfatases in mice and humans. $J \mathrm{Bio} / \mathrm{Chem}$ 2002, 277:49175-49185.

18. Ohto T, Uchida H, Yamazaki H, Keino-Masu K, Matsui A, Masu M: Identification of a novel nonlysosomal sulphatase expressed in the floor plate, choroid plexus and cartilage. Genes Cells 2002, 7:173-185.

19. Ai X, Do AT, Lozynska O, Kusche-Gullberg M, Lindahl U, Emerson CP Jr: QSulf1 remodels the 6-O sulfation states of cell surface heparan sulfate proteoglycans to promote Wnt signaling. J Cell Biol 2003, 162:341-351.

20. Nawroth R, van Zante A, Cervantes S, McManus M, Hebrok M, Rosen SD: Extracellular sulfatases, elements of the Wnt signaling pathway, positively regulate growth and tumorigenicity of human pancreatic cancer cells. PLOS ONE 2007, 2:e392.

21. Narita K, Chien J, Mullany SA, Staub J, Qian X, Lingle WL, Shridhar $V$ : Loss of HSulf-1 expression enhances autocrine signaling mediated by amphiregulin in breast cancer. J Biol Chem 2007, 282:14413-14420.

22. Holst CR, Bou-Reslan $H$, Gore BB, Wong $K$, Grant D, Chalasani S, Carano RA, Frantz GD, Tessier-Lavigne M, Bolon B, French DM, Ashkenazi A: Secreted sulfatases Sulf1 and Sulf2 have overlapping yet essential roles in mouse neonatal survival. PLoS ONE 2007, 2:e575.

23. Ai X, Kitazawa T, Do AT, Kusche-Gullberg M, Labosky PA, Emerson CP Jr: SULF1 and SULF2 regulate heparan sulfate-mediated GDNF signaling for esophageal innervation. Development 2007, 134:3327-3338.

24. Wang S, Ai X, Freeman SD, Pownall ME, Lu Q, Kessler DS, Emerson CP Jr: QSulf1, a heparan sulfate 6-O-endosulfatase, inhibits fibroblast growth factor signaling in mesoderm induction and angiogenesis. Proc Natl Acad Sci USA 2004, 101:4833-4838. 
25. Thomas CM, Fuller CJ, Whittles CE, Sharif M: Chondrocyte death by apoptosis is associated with cartilage matrix degradation. Osteoarthritis Cartilage 2007, 15:27-34.

26. Xu L, Peng H, Glasson S, Lee PL, Hu K, ljiri K, Olsen BR, Goldring $\mathrm{MB}, \mathrm{Li}$ Y: Increased expression of the collagen receptor discoidin domain receptor 2 in articular cartilage as a key event in the pathogenesis of osteoarthritis. Arthritis Rheum 2007, 56:2663-2673.

27. Blanco FJ, Ochs RL, Schwarz H, Lotz M: Chondrocyte apoptosis induced by nitric oxide. Am J Pathol 1995, 146:75-85.

28. Staub J, Chien J, Pan Y, Qian X, Narita K, Aletti G, Scheerer M, Roberts LR, Molina J, Shridhar V: Epigenetic silencing of HSulf1 in ovarian cancer: implications in chemoresistance. Oncogene 2007, 26:4969-4978.

29. Guilak F, Alexopoulos LG, Upton ML, Youn I, Choi JB, Cao L, Setton LA, Haider MA: The pericellular matrix as a transducer of biomechanical and biochemical signals in articular cartilage. Ann N Y Acad Sci 2006, 1068:498-512.

30. Uchimura K, Morimoto-Tomita M, Bistrup A, Li J, Lyon M, Gallagher J, Werb Z, Rosen SD: HSulf-2, an extracellular endoglucosamine-6-sulfatase, selectively mobilizes heparin-bound growth factors and chemokines: effects on VEGF, FGF-1, and SDF-1. BMC Biochem 2006, 7:2.

31. Morimoto-Tomita M, Uchimura K, Bistrup A, Lum DH, Egeblad M, Boudreau N, Werb Z, Rosen SD: Sulf-2, a proangiogenic heparan sulfate endosulfatase, is upregulated in breast cancer. Neoplasia 2005, 7:1001-1010.

32. Roman-Blas JA, Stokes DG, Jimenez SA: Modulation of TGFbeta signaling by proinflammatory cytokines in articular chondrocytes. Osteoarthritis Cartilage 2007, 15:1367-1377.

33. Westacott Cl, Sharif M: Cytokines in osteoarthritis: mediators or markers of joint destruction? Semin Arthritis Rheum 1996, 25:254-272.

34. Cecil DL, Johnson K, Rediske J, Lotz M, Schmidt AM, Terkeltaub $\mathrm{R}$ : Inflammation-induced chondrocyte hypertrophy is driven by receptor for advanced glycation end products. J Immunol 2005, 175:8296-8302.

35. Hashimoto $S$, Setareh M, Ochs RL, Lotz M: Fas/Fas ligand expression and induction of apoptosis in chondrocytes. Arthritis Rheum 1997, 40:1749-1755.

36. Esko JD, Selleck SB: Order out of chaos: assembly of ligand binding sites in heparan sulfate. Annu Rev Biochem 2002, 71:435-471.

37. Lum DH, Tan J, Rosen SD, Werb Z: Gene trap disruption of the mouse heparan sulfate 6-0-endosulfatase gene, Sulf2. Mol Cell Biol 2007, 27:678-688.

38. Flannery CR, Hughes CE, Schumacher BL, Tudor D, Aydelotte $\mathrm{MB}$, Kuettner KE, Caterson B: Articular cartilage superficial zone protein (SZP) is homologous to megakaryocyte stimulating factor precursor and is a multifunctional proteoglycan with potential growth-promoting, cytoprotective, and lubricating properties in cartilage metabolism. Biochem Biophys Res Commun 1999, 254:535-541.

39. Schumacher BL, Hughes CE, Kuettner KE Caterson B, Aydelotte $M B$ : Immunodetection and partial CDNA sequence of the proteoglycan, superficial zone protein, synthesized by cells lining synovial joints. J Orthop Res 1999, 17:110-120.

40. Schmidt TA, Schumacher BL, Klein TJ, Voegtline MS, Sah RL: Synthesis of proteoglycan 4 by chondrocyte subpopulations in cartilage explants, monolayer cultures, and resurfaced cartilage cultures. Arthritis Rheum 2004, 50:2849-2857.

41. Gepstein A, Arbel G, Blumenfeld I, Peled M, Livne E: Association of metalloproteinases, tissue inhibitors of matrix metalloproteinases, and proteoglycans with development, aging, and osteoarthritis processes in mouse temporomandibular joint. Histochem Cell Biol 2003, 120:23-32.

42. Plaas AH, West LA, Wong-Palms S, Nelson FR: Glycosaminoglycan sulfation in human osteoarthritis. Disease-related alterations at the non-reducing termini of chondroitin and dermatan sulfate. J Biol Chem 1998, 273:12642-12649.

43. Okabe T, Ohmori $Y$, Tanigami A, Hishigaki H, Suzuki $Y$, Sugano $S$, Kawaguchi $A$, Nakaya $H$, Wakitani $S$ : Detection of gene expression in synovium of patients with osteoarthritis using a random sequencing method. Acta Orthop 2007, 78:687-692.

44. Patterson AM, Cartwright A, David G, Fitzgerald O, Bresnihan B, Ashton BA, Middleton J: Differential expression of syndecans and glypicans in chronically inflamed synovium. Ann Rheum Dis 2008, 67:592-601.

45. Salminen-Mankonen $\mathrm{H}$, Saamanen AM, Jalkanen M, Vuorio E, Pirila $L$ : Syndecan-1 expression is upregulated in degenerating articular cartilage in a transgenic mouse model for osteoarthritis. Scand J Rheumatol 2005, 34:469-474.

46. Pfander D, Swoboda B, Kirsch T: Expression of early and late differentiation markers (proliferating cell nuclear antigen, syndecan-3, annexin $\mathrm{VI}$, and alkaline phosphatase) by human osteoarthritic chondrocytes. Am J Pathol 2001, 159:1777-1783.

47. Barre PE, Redini F, Boumediene K, Vielpeau C, Pujol JP: Semiquantitative reverse transcription-polymerase chain reaction analysis of syndecan -1 and -4 messages in cartilage and cultured chondrocytes from osteoarthritic joints. Osteoarthritis Cartilage 2000, 8:34-43.

48. Krenn V, Hensel F, Kim HJ, Souto Carneiro MM, Starostik P, Ristow G, Konig A, Vollmers HP, Muller-Hermelink HK: Molecular $\operatorname{lgV}(\mathrm{H})$ analysis demonstrates highly somatic mutated $\mathrm{B}$ cells in synovialitis of osteoarthritis: a degenerative disease is associated with a specific, not locally generated immune response. Lab Invest 1999, 79:1377-1384.

49. Imai S, Kaksonen M, Raulo E, Kinnunen T, Fages C, Meng X, Lakso $\mathrm{M}$, Rauvala $\mathrm{H}$ : Osteoblast recruitment and bone formation enhanced by cell matrix-associated heparin-binding growthassociated molecule (HB-GAM). J Cell Biol 1998, 143:1113-1128

50. Tesche F, Miosge N: Perlecan in late stages of osteoarthritis of the human knee joint. Osteoarthritis Cartilage 2004, 12:852-862

51. Hayes AJ, Tudor D, Nowell MA, Caterson B, Hughes CE: Chondroitin sulfate sulfation motifs as putative biomarkers for isolation of articular cartilage progenitor cells. J Histochem Cytochem 2008, 56:125-138.

52. Rodgers KD, Sasaki T, Aszodi A, Jacenko O: Reduced perlecan in mice results in chondrodysplasia resembling SchwartzJampel syndrome. Hum Mol Genet 2007, 16:515-528.

53. Tesche F, Miosge N: New aspects of the pathogenesis of osteoarthritis: the role of fibroblast-like chondrocytes in late stages of the disease. Histol Histopathol 2005, 20:329-337.

54. Knudson $\mathrm{CB}$, Knudson W: Cartilage proteoglycans. Semin Cell Dev Biol 2001, 12:69-78.

55. Habuchi $\mathrm{H}$, Habuchi $\mathrm{O}$, Kimata $\mathrm{K}$ : Sulfation pattern in glycosaminoglycan: does it have a code? Glycoconj J 2004, 21:47-52.

56. Lundin L, Larsson H, Kreuger J, Kanda S, Lindahl U, Salmivirta M, Claesson-Welsh L: Selectively desulfated heparin inhibits fibroblast growth factor-induced mitogenicity and angiogenesis. J Biol Chem 2000, 275:24653-24660.

57. Im HJ, Muddasani $P$, Natarajan V, Schmid TM, Block JA, Davis F, van Wijnen AJ, Loeser RF: Basic fibroblast growth factor stimulates matrix metalloproteinase-13 via the molecular cross-talk between the mitogen-activated protein kinases and protein kinase Cdelta pathways in human adult articular chondrocytes. J Biol Chem 2007, 282:11110-11121.

58. Hwang SG, Ryu JH, Kim IC, Jho EH, Jung HC, Kim K, Kim SJ, Chun JS: Wnt-7a causes loss of differentiated phenotype and inhibits apoptosis of articular chondrocytes via different mechanisms. J Biol Chem 2004, 279:26597-26604.

59. Yates KE, Shortkroff S, Reish RG: Wnt influence on chondrocyte differentiation and cartilage function. DNA Cell Biol 2005, 24:446-457. 